Präv Gesundheitsf 2021 · 16:249-255

https://doi.org/10.1007/s11553-020-00819-4

Eingegangen: 10 . August 2020

Angenommen: 9. November 2020

Online publiziert: 3. Dezember 2020

(c) Der/die Autor(en) 2020

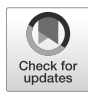

Ilona Efimov $(\mathbb{D} \cdot$ - Volker Harth · Stefanie Mache

Zentralinstitut für Arbeitsmedizin und Maritime Medizin (ZfAM), Universitätsklinikum Hamburg-

Eppendorf (UKE), Hamburg, Deutschland

\title{
Gesundheitsförderung in virtueller Teamarbeit durch gesundheitsorientierte Führung
}

Welche Auswirkungen eine fortschreitende Digitalisierung des Arbeitsplatzes auf die Gesundheit der Beschäftigten hat, verdeutlichen Befragungsergebnisse mit über 8019 Beschäftigten: Insgesamt $23 \%$ der Befragten gaben an, emotional erschöpft zu sein [3].

In dieser sich verändernden Arbeitswelt entstehen neue flexible Arbeitsformen wie die virtuelle Teamarbeit. Hierunter werden räumlich verteilte Teams verstanden, welche hauptsächlich über digitale Informations- und Kommunikationstechnologien(IKT) kommunizieren und dabei über einen bestimmten Zeitraum an gemeinsamen Zielen und Aufgaben arbeiten [2, 22, 28].

Die flexiblen Arbeitsbedingungen in virtuellen Teams verändern folglich die Anforderungen und Ressourcen der Beschäftigten. Auf der einen Seite kann die Flexibilität zu einer verbesserten Work-Life-Balance und somit Arbeitszufriedenheit führen [28]. Neben den flexiblen Arbeitszeiten kann u. a. auch die soziale Unterstützung, z. B. in Form von Zielentwicklungen, der Förderung der Teamkultur oder dem sozialen Austausch mit der direkten Führungskraft sowie Kolleg*innen, eine wichtige Ressource darstellen. Auch die durch virtuelle Teamarbeit entstandenen Handlungsund Entscheidungsspielräume können sich als Ressourcen positiv auf die Motivation von virtuellen Teammitgliedern und Führungskräften auswirken [22]. Hingegen können irreguläre Arbeitszeiten, Fehlerquellen in der Kommunikation, eine erhöhte Konfliktanfälligkeit und eine erschwerte Integration neuer Teammitglieder als psychische Belas- tungsfaktoren erlebt werden [22]. Der eingeschränkte oder fehlende persönliche Kontakt bei den virtuellen Teammitgliedern kann dabei zu Entgrenzung, Rollenunklarheit und Vertrauens- und Motivationsverlust führen [42]. Zudem können nach aktueller Studienlage auch durch die Nutzung von digitaler IKT psychische Belastungsfaktoren entstehen, welche gesundheitliche Folgen für die Beschäftigten haben können. Hierzu zählen Beschleunigung, Komplexität, fehlende Benutzerfreundlichkeit, Unterbrechungen, eine Omni- und Dauerpräsenz, ein Kommunikationsrauschen durch IKT sowie eine Unzuverlässigkeit durch technische Probleme [29]. Die Veränderungen der flexibilisierten Arbeitswelt beeinflussen auch die Einflussmöglichkeiten von Führung [40] und stellen erhöhte Anforderungen an virtuelle Führungskräfte $[1,5,16,34$, 42].

Die vorliegende Übersichtsarbeit legt den Fokus auf die gesundheitsorientierte Führung in virtueller Teamarbeit, da diese flexible Arbeitsform in Organisationen weltweit verstärkt Anwendung findet [38] und von erhöhten gesundheitlichen Belastungsfaktoren gekennzeichnet ist [29]. Führungsspezifische Einflussmöglichkeiten sollen hierbei aufgezeigt werden und der Ansatz gesundheitsorientierter Führung differenziert beleuchtet werden.

\section{Führung als Gesundheitsfaktor}

Die Übersichtsarbeit zum empirischen Zusammenhang zwischen Führungsverhalten und Mitarbeitergesundheit von psychischer Gesundheit am Arbeitsplatz
für Individuen als auch Organisationen. 
Gregersen et al. [15] zeigt, dass Führung sowohl als Stressor als auch als Ressource auf Mitarbeitende wirken kann. Zahlreiche Studien haben zudem den Einfluss von Führung auf die Gesundheit und das Wohlbefinden von abhängig Beschäftigten ohne Führungsverantwortung nachweisen können [15, 27, 31, 32, 41, 45]. Die aktuelle Metaanalyse von Kaluza et al. [18] konnte aufzeigen, dass auch das Wohlbefinden der Führungskräfte einen entscheidenden Einfluss auf den Zusammenhang von Führungsverhalten und Beschäftigtengesundheit haben kann.

Nach Franke et al. [11] bestehen vier Wirkmechanismen, wie Führung einen Einfluss auf die Gesundheit der Mitarbeitenden haben kann. Zum einen kann die Führungskraft durch ihr Verhalten und die Art der Kommunikation die Gesundheit der Mitarbeitenden direkt beeinflussen. Zum anderen kann auch ein indirekter Einfluss über die Gestaltung der Arbeitsbedingungen erfolgen. Aufgrund der spezifischen Arbeitsanforderungen einer Führungskraft können auch Führungskräfte selbst psychischen Belastungsfaktoren ausgesetzt sein. Das Stresserleben kann dabei ein Gesundheitsrisiko für die Führungskräfte selbst als auch für ihre unterstellten Mitarbeitenden bedeuten. Zuletzt fungieren Führungskräfte als Vorbilder für Mitarbeitende und dienen als Multiplikatoren für das Betriebliche Gesundheitsmanagement [11]. So kann die Einstellung von Mitarbeitenden durch die Einstellung ihrer Führungskraft beeinflusst werden [33], ebenso kann die Wahrscheinlichkeit erhöht werden, Arbeitssicherheitspraktiken bei einem positiven Vorbild anzuwenden [6].

Bereits etablierte Führungskonzepte wurden vielfach in Bezug zur Gesundheit der Beschäftigten empirisch untersucht. $\mathrm{Zu}$ gesundheitsrelevanten Führungskonzepten im virtuellen Arbeitskontext zählen die transformationale Führung und die beziehungs- und aufgabenorientierte Führung [42]. Metaanalysen ergaben, dass ein transformationaler sowie ein mitarbeiterorientierter Führungsstil positive gesundheitliche Effekte, wie geringe gesundheitliche Beschwerden, eine Reduzierung von Stress und Burnout und einer Steigerung von Wohl- befinden und Arbeitszufriedenheit auf die Beschäftigten haben [15, 31]. Diese Führungskonzepte wurden jedoch nicht entwickelt, um vorrangig gesundheitsrelevantes Führungsverhalten vorherzusagen, sondern Leistung [44]. Da sich von diesen klassischen Führungskonzepten wenige konkrete Handlungsempfehlungen und Maßnahmen für die Praxis ableiten lassen, wurden spezifischere Konzepte gesunder Führung entwickelt [12].

\section{Gesundheitsorientierte Führung}

\section{Übersicht zu Konzepten gesunder Führung}

Eine aktuelle systematische Literaturrecherche macht deutlich, dass in den letzten 2 Jahrzehnten bereits einige theoretische Konzepte und empirische Studien zu gesunder Führung parallel entstanden sind, die sich in vielerlei Hinsicht überschneiden, aber auch voneinander abgrenzen lassen [37]. Auch wenn aufgrund der Unterschiede hinsichtlich der Methodenwahl und Forschungszwecke keine absoluten Vergleiche möglich sind, so konnten inhaltliche Gemeinsamkeiten zwischen den theoretischen Konzepten festgestellt werden. Es besteht Einigkeit darüber, dass es beobachtbare Unterschiede in dem gesundheitsförderlichen Verhalten, den Werten und Einstellungen von Führungskräften gibt. Weiterhin wurde die Relevanz von gesunder Führung für die betriebliche Gesundheitsförderung herausgestellt und als Bindeglied zwischen individueller Gesundheit und betrieblicher Gesundheitsförderung erkannt. Die Übersichtsarbeit identifizierte zwischen allen theoretischen Konzepten zwei dominante Konzepte gesunder Führung: die gesundheitsförderliche („health-promoting leadership“) und die gesundheitsorientierte („health-oriented leadership“) Führung [37]. Eine gesundheitsförderliche Führung kann nach Eriksson et al. [9] anhand dreier Komponenten definiert werden: ein unterstützender Führungsstil, die Organisation gesundheitsförderlicher Aktivitäten sowie die Bereitstellung eines gesundheitsförderlichen Arbeitsplatzes.
Im Vergleich zur gesundheitsförderlichen Führung zeigt sich, dass der HoL-Ansatz der gesundheitsorientierten Führung („health-oriented leadership“) in einem breiten deduktiven Prozess entwickelt wurde und auch die Faktorenstruktur bestätigt werden konnte [12]. Alle empirischen quantitativen Studien zur gesundheitsorientierten Führung verwenden zudem das für diesen Führungsansatz entwickelte Diagnoseinstrument [37], welches in deutscher und englischer Sprache erarbeitet wurde $[12,14]$. Die Struktur und Validität des Instruments konnte durch weitere praxisrelevante Studien bestätigt werden [14, $17,39]$. Die Studien zur gesundheitsförderlichen Führung hingegen verwenden kein einheitliches Diagnoseinstrument [37]. Weiterhin konnte im Unterschied zur gesundheitsförderlichen Führung die Selbstführung der Führungskraft im Ansatz gesundheitsorientierter Führung berücksichtigt werden [11].

Die Kritik der systematischen Literaturrecherche von Rudolph et al. [37] weist Forscher*innen jedoch darauf hin, dass nach dem aktuellem Stand der Forschung die bestehenden Konstrukte differenzierter untersucht werden müssen, u. a. auch die inkrementelle Validität. Erst dann lassen sich spezifische Ansätze gesunder Führung von klassischen Führungskonzepten abgrenzen.

\section{Definition des HoL-Ansatzes}

Der integrative HoL-Ansatz nach Franke und Felfe [12] kombiniert alle vier Wirkmechanismen von Führung und schließt neben der klassischen Mitarbeiterführung auch die gesundheitsorientierte Selbstführung sowohl der Führungskraft als auch der Beschäftigten ein $[12,14]$. Gesundheitsorientierte Führung wird hierbei nicht als ein klar abgrenzbares Führungskonzept verstanden, sondern als ein Führungsansatz, welcher nur einen spezifischen Teilbereich des Führungsverhaltens fokussiert [42].

Das HoL-Modell besteht aus drei Komponenten (SelfCare-Führungskraft, StaffCare und SelfCare-Mitarbeitende), wobei die gesundheitsorientierte Selbstführung der Führungskraft (SelfCare) 
die Grundlage bildet. Alle drei Komponenten setzen sich aus jeweils drei Dimensionen zusammen: Wichtigkeit, Achtsamkeit und Verhalten. Um sich selbst gesundheitsorientiert führen $\mathrm{zu}$ können, muss die eigene Gesundheit als wichtig eingestuft werden, die persönlichen Belastungsfaktoren am Arbeitsplatz müssen in den entsprechenden Situationen wahrgenommen werden und zuletzt muss auch Handlungsfähigkeit bestehen, um das eigene Gesundheitsbewusstsein in ein entsprechendes gesundheitsförderliches und -präventives Verhalten zu übersetzen [11].

Auch hinsichtlich der StaffCare nimmt das Modell an, dass im ersten Schritt die Gesundheit der Mitarbeitenden durch die jeweilige Führungskraft als wichtig eingestuft werden muss. Im zweiten Schritt muss eine Achtsamkeit für das Gesundheitsbefinden der Mitarbeitenden bestehen, damit im letzten Schritt entsprechendes gesundheitsorientiertes Führungsverhalten gezeigt werden kann. Die SelfCare der Führungskraft ist hierbei für das Modell grundlegend, denn sie ermöglicht zum einen eine gesundheitsorientierte Mitarbeiterführung (StaffCare) und zum anderen kann eine ausgeprägte SelfCare als Rollenmodell für Mitarbeitende dienen und die eigene gesundheitsorientierte Selbstführung (SelfCare) der Mitarbeitenden positiv beeinflussen. Das heuristische Modell des Führungseinflusses auf die Gesundheit der Mitarbeitenden nimmt dabei an, dass die SelfCare der Führungskraft die SelfCare der Mitarbeitenden positiv über die StaffCare beeinflusst. Eine gesundheitsorientierte Mitarbeiter- und Selbstführung der Führungskraft wie auch der Mitarbeitenden erhöhen dabei die Wahrscheinlichkeit positiver Gesundheitsparameter in der Belegschaft wie ein erhöhtes Wohlbefinden oder reduziertes Stresserleben [11].

\section{Aktueller Stand der Forschung}

Nach dem aktuellen Stand der Forschung bestehen bislang nur 8 quantitative Studien $[13,14,17,20,21,25,26,39]$, die konkret den Ansatz und das Instrument der gesundheitsorientierten Führung nach Franke und Felfe [12] empi-

Präv Gesundheitsf 2021 · 16:249-255 https://doi.org/10.1007/s11553-020-00819-4

(c) Der/die Autor(en) 2020

\section{Gesundheitsförderung in virtueller Teamarbeit durch gesundheitsorientierte Führung}

\section{Zusammenfassung}

Hintergrund. Virtuelle Teamarbeit als neue flexible Arbeitsform ist durch spezifische Herausforderungen und damit einhergehenden gesundheitlichen Belastungsfaktoren gekennzeichnet. Der Einfluss von Führungskräften auf die Gesundheit von Mitarbeitenden konnte bereits im direkten und persönlichen Teamkontakt nachgewiesen werden.

Ziel der Arbeit. Der Artikel soll eine Übersicht zu Konzepten gesunder Führung bieten und diese im virtuellen Arbeitskontext beleuchten.

Material und Methoden. In einer explorativen Literaturrecherche werden bisher verfügbare Erkenntnisse zu Konzepten gesunder Führung recherchiert und zusammengefasst. Aus diesen Forschungserkenntnissen wird ein geeigneter Ansatz gesunder Führung identifiziert und der empirische Forschungsstand in Bezug zur virtuellen Teamarbeit dargestellt. Ergebnisse. Diese Übersichtsarbeit identifizierte zwischen allen theoretischen
Konzepten gesunder Führung die gesundheitsorientierte Führung als einen dominanten Ansatz. Die zentralen Ergebnisse bisheriger Studien zeigen auf, dass gesundheitsorientierte Führung einen signifikant positiven Einfluss auf die Gesundheit von Beschäftigten haben kann. Insgesamt stellt Führung einen relevanten Gesundheitsfaktor für die Betriebliche Gesundheitsförderung dar.

Schlussfolgerung. Der empirische Forschungsstand verdeutlicht, dass noch Forschungsbedarf hinsichtlich gesundheitsorientierter Führung besteht. Die Relevanz weiterer empirischer Studien für den virtuellen Arbeitskontext wird für die Wissenschaft und Praxis diskutiert.

Schlüsselwörter

Gesunde Führung · Virtuelle Teams · Virtuelle Führung · Führungseinfluss · Betriebliches Gesundheitsmanagement

\section{Health promotion in virtual teamwork through health-oriented leadership}

\section{Abstract}

Background. Virtual teamwork as a new way of working is characterized by specific challenges and associated job demands. Leadership influence on the health of employees has already been proven in direct and personal team constellations.

Objectives. The goal of this article is to provide an overview of healthy leadership concepts and illustrate them in a virtual working environment.

Materials and methods. In an explorative literature review, currently available findings on healthy leadership concepts are evaluated and summarized. Based on this, a suitable approach to healthy leadership is identified and the empirical state of research in virtual teamwork is presented.

Results. Our review identified health-oriented leadership as the dominant approach among all theoretical concepts of healthy leadership. The central results of previous studies show that health-oriented leadership can have a significantly positive influence on the health of employees. Overall, leadership represents a relevant health factor for workplace health promotion.

Conclusions. The empirical state of research shows that there is still much need for research on health-oriented leadership in virtual teamwork. The relevance of further empirical studies for virtual work environments is discussed for science and practice.

Keywords Healthy leadership - Virtual teams · Virtual leadership - Leadership influence - Occupational health management 
risch untersucht haben. Erste empirische Ergebnisse bestätigten die Grundannahmen, die in diesem Führungsansatz getroffen wurden [13, 14]. Mitarbeitende wiesen höhere Ausprägungen hinsichtlich der Achtsamkeit und dem Verhalten ihrer eigenen SelfCare auf und berichteten von einem besseren Gesundheitszustand sowie weniger Irritationen, Gesundheitsbeschwerden und Konflikten zwischen der Familie und dem Arbeitsplatz, wenn sie wahrnehmen konnten, dass ihre Führungskraft gesundheitsorientiertes Verhalten gezeigt hat [14]. Es konnte gezeigt werden, dass die Gesundheit der Belegschaft nicht nur von der gesundheitsorientierten Mitarbeiterführung abhängt, sondern auch von der gesundheitsorientierten Selbstführung der Mitarbeitenden. Dieses Ergebnis macht deutlich, dass Mitarbeitende auch eine aktive Rolle im Zusammenhang von Führung und Gesundheit am Arbeitsplatz spielen [14].

Führungskräfte können zudem einen indirekten Einfluss auf den Gesundheitszustand der Mitarbeitenden über ihre gesundheitsorientierte Selbstführung haben. In 2 Studien von Kranabetter und Niessen [25] waren Mitarbeitende weniger erschöpft und zynisch, wenn transformationale Führungskräfte ein Bewusstsein für ihren eigenen Gesundheitszustand entwickelt haben. In ihrer Studie zeigte sich zudem, dass Mitarbeitende auch weniger erschöpft waren, wenn Führungskräfte mit einem transformationalen Führungsstil gesundheitsorientiertes Verhalten gezeigt haben. Führungskräfte können somit hinsichtlich ihrer Achtsamkeit und ihres Gesundheitsverhaltens eine Vorbildfunktion für ihre Mitarbeitenden einnehmen [25]. Neben der gesundheitsorientierten Selbstführung zeigten Köppe et al. [21], dass der Gesundheitszustand der Führungskraft selbst einen indirekten Crossover-Effekt auf den Gesundheitszustand der Mitarbeitenden haben kann. In ihrer Studie hatte der Erschöpfungszustand der Führungskraft über ihre gesundheitsorientierte Mitarbeiterführung einen indirekten Einfluss auf die somatischen Beschwerden der Mitarbeitenden [21].
Welchen entscheidenden Einfluss die Kombination aller drei Komponenten des HoL-Ansatzes (StaffCare, SelfCare der Führungskräfte und Mitarbeitenden) auf die Gesundheit der Mitarbeitenden haben können, konnten Klug et al. [20] nachweisen. Sie konnten erstmalig anhand des HoL-Ansatzes vier Profile gesundheitsorientierter Führung identifizieren und diese auch im Zusammenhang mit Gesundheitsparametern untersuchen. Die konsistenten Profile zeichnen sich durch ausschließlich positive („high care“) oder negative („low care") Ausprägungen der drei Komponenten StaffCare-, SelfCare-Führungskraft und SelfCare-Mitarbeitende aus. Zwei inkonsistente Profile werden durch Diskrepanzen in den Ausprägungen von Selbst- oder Mitarbeiterführung („leader sacrifice" und „follower sacrifice") beschrieben. Die Studienergebnisse zeigen, dass insbesondere das „High-care-Pro-

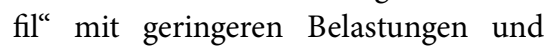
besserer Gesundheit der Mitarbeitenden korreliert. Dieses Profil zeichnet sich durch hohe Ausprägungen in der StaffCare, der SelfCare der Führungskraft und der SelfCare der Mitarbeitenden sowie niedrigen Ausprägungen im gesundheitsgefährdenden Verhalten aus [20].

Des Weiteren kann eine gesundheitsorientierte Führung das gesundheitsförderliche Verhalten von Mitarbeitenden positiv beeinflussen [26]. In der Studie von Krick et al. [26] wurde ersichtlich, dass neben organisationalen und individuellen Faktoren auch die gesundheitsorientierte Selbst- und Mitarbeiterführung der Führungskräfte die Wahrscheinlichkeit erhöhen, dass Mitarbeitende nicht nur die Intention haben, sondern auch tatsächlich an Kursen betrieblicher Gesundheitsförderung teilnehmen.

Bislang sind zwei quantitative Studien zum HoL-Ansatz in zwei spezifischen Branchen in Deutschland durchgeführt worden, die beide besondere BelastungsBeanspruchungssituationen für die $\mathrm{Be}$ legschaft belegen [17, 39]. Die erste branchenspezifische Studie ist in der stationären Altenpflege durchgeführt worden, einer Branche, die von steigenden Belastungen der Mitarbeitenden und einer hohen Personalfluktuation gekennzeichnet ist [17]. Die Studienergebnisse verdeutlichen, dass ein positiver direkter Zusammenhang zwischen gesundheitsorientierter Führung und der Gesundheit sowie dem organisationalen Commitment der Mitarbeitenden besteht, welcher durch soziale Ressourcen und Belastungen teilweise mediiert wird. Es wird vermutet, dass die emotionale Einstellung der Mitarbeitenden gegenüber dem Unternehmen durch die Gesundheitsorientierung ihrer Führungskraft beeinflusst wird, da sie als eine generelle Unterstützung wahrgenommen werden kann. Die Mediation über die sozialen Kontextfaktoren zeigt, dass gesundheitsorientierte Führungskräfte auch die sozialen Beziehungen und das soziale Klima im Team beeinflussen können [17]. Die zweite branchenspezifische Studie wurde bei der Polizei durchgeführt. Der Arbeitsplatz zeichnet sich durch unterschiedliche betriebliche und organisatorische Belastungsfaktoren aus [39]. Die Studienergebnisse zeigen, dass gesundheitsorientierte Führung das Wohlbefinden der Mitarbeitenden sowohl direkt als auch indirekt über die SelfCare der Mitarbeitenden positiv beeinflusst. Weiterhin konnte ein negativer Zusammenhang zwischen gesundheitsorientierter Führung und hohen Ausprägungen von Burnout, Depression und körperlichen Beschwerden festgestellt werden [39].

Studien zur gesundheitsorientierten Führung, welche nicht das HoL-Instrument verwendet haben, liefern darüber hinaus weiterführende Erkenntnisse zur Wirkweise des Führungsansatzes. Eine qualitative Interviewstudie mit Führungskräften identifizierte Verhaltensweisen, wie mit erschöpften Mitarbeitenden umgegangen werden kann. $\mathrm{Zu}$ den effektivsten Verhaltensweisen in diesem Zusammenhang zählen die (Neu-)Gestaltung von Aufgaben, die emotionale Unterstützung, die Kommunikation von Erwartungen und Hilfsangebote durch die Führungskraft [24].

Weiterhin zeigen die Ergebnisse einer internationalen Längsschnittstudie mit über 2316 Beschäftigten und ihren 245 Führungskräften aus Deutschland, Finnland und Schweden, dass gesund- 
heitsorientierte Führung neben vier weiteren untersuchten Führungsansätzen die größte Vorhersagekraft in der Erklärung einer nachhaltigen Verbesserung mehrerer Gesundheitsmerkmale aufweist [35]. Die Wahrnehmung gesundheitsorientierter Führung geht über alle drei Messzeitpunkte mit einem gesteigerten Commitment der Beschäftigten und einer Reduzierung von Depressivität einher. Langfristig zeigte sich zudem, dass eine gesundheitsorientierte Führung zu einer Senkung emotionaler Erschöpfung und somatischer Beschwerden, einer Verbesserung des Teamklimas und einer Erhöhung des Arbeitsengagements der Beschäftigten führt. Zudem wurden Wechselwirkungen zwischen gesundheitsorientierter Führung und der Einschätzung von Belastungen (reduzierte Arbeitsplatzunsicherheit) und Ressourcen (erhöhte Autonomie und Sinnerleben) am Arbeitsplatz festgestellt. Führungskräfte haben somit auch indirekt einen Einfluss auf die Beschäftigtengesundheit, indem sie die Gestaltung der Arbeitsbedingungen beeinflussen können. Die Ergebnisse verdeutlichen, dass dieser Führungsansatz Handlungsspielräume und Partizipation am Arbeitsplatz ermöglichen kann [35].

\section{Gesundheitsorientierte Führung in virtuellen Teams}

Ein Übersichtsartikel zur gesundheitsorientierten Führung im Kontext flexibler Arbeitswelten macht deutlich, dass orts- und zeitunabhängige Arbeit sowohl für Mitarbeitende als auch für Führungskräfte eine besondere Herausforderung darstellt und insbesondere die Anforderungen an Kommunikationskompetenzen erhöht [36]. Eine spezifische Form flexibler Arbeit stellt die virtuelle Teamarbeit dar. Kordsmeyer et al. [23] stellten in ihrem Übersichtsartikel heraus, dass gesundheitsorientierte Führung in virtuellen Teams spezifischen Anforderungen unterliegt. Über die allgemeinen Führungsaufgaben in virtuellen Teams hinaus sollten Führungskräfte demnach im Sinne einer gesundheitsorientierten Führung auf potenzielle Gesundheitsrisiken wie der erweiterten arbeitsbezogenen Erreich- barkeit, den Konflikten bei der Vereinbarkeit von Arbeit und Privatleben und dem geringeren sozialen Austausch im Team achten [23]. Basierend auf dem Konzept der gesundheitsorientierten Führung nach Franke et al. [11] wurden zudem Interventionsmöglichkeiten zur Förderung der Mitarbeiter- und Selbstführung von Führungskräften sowie Teammitgliedern entwickelt. Führungskräfte sollten demnach im Sinne einer gesundheitsorientierten Mitarbeiterführung beispielsweise Kick-off-Veranstaltungen zu Beginn der Zusammenarbeit initiieren, Regelungen bezüglich erweiterter Erreichbarkeit vereinbaren und Maßnahmen zur Vertrauensförderung und Instrumenten der (Online-)Gefährdungsbeurteilung umsetzen. Chatforen oder virtuelle Kaffeepausen können zudem den sozialen Austausch fördern [23]. Führungskräfte sollten diese Form des informellen Austauschs fördern oder selbst initiieren. Auf diese Weise können beispielsweise soziale Kontextfaktoren wie unterschiedliche Feiertage in multikulturellen Teams thematisiert und gegenseitiges Verständnis gefördert werden [4].

Nach dem aktuellen Stand der Forschung existieren bislang nur die Ergebnisse zweier qualitativen Studien zu gesunder Führung in virtueller Teamarbeit [7]. In der ersten Interviewstudie wurden virtuelle Führungskräfte zu ihrem Einfluss auf die gesundheitsförderlichen bzw. -belastenden Arbeitsbedingungen von Mitarbeitenden befragt. Zwar erkannten die Führungskräfte den Einfluss psychischer Ursachen auf ihre physische Gesundheit, jedoch stellten die wenigsten einen Zusammenhang zwischen Führungsverhalten und Beschäftigtengesundheit her. Stattdessen erkannten sie einen Einfluss ihres Führungsverhaltens auf das Betriebsklima und die Unternehmenskultur. Zudem verdeutlichen die Befragungsergebnisse, dass sich die virtuellen Führungskräfte der spezifischen psychischen Belastungen am Arbeitsplatz bewusst sind und diesen mit eigens entwickelten Ausgleichsaktivitäten begegnen [7]. Die zweite Interviewstudie hat unter Anwendung des Ansatzes gesundheitsorientierter Führung nach Franke und Felfe [12] virtuelle Füh- rungskräfte zu ihrer gesundheitsorientierten Selbst- und Mitarbeiterführung befragt [8]. Die Ergebnisse konnten aufzeigen, dass virtuelle Führungskräfte sowohl in der gesundheitsorientierten Selbstführung als auch in der gesundheitsorientierten Mitarbeiterführung der Gesundheit eine hohe Wichtigkeit zuschreiben und achtsam gegenüber dem Gesundheitszustand sind. Um sich selbst gesundheitsorientiert zu führen, geben die Führungskräfte insbesondere körperliche Betätigung und ihr Grenzziehungsverhalten zur Arbeit an. Für eine gesundheitsorientierte Mitarbeiterführung werden mehrheitlich die Kommunikation, der Vertrauensaufbau, die Unterstützung in der Grenzziehung zur Arbeit und die Umsetzung persönlicher Treffen beschrieben. Neben sozialen, technischen und persönlichen Faktoren werden vornehmlich organisationale Einflussfaktoren in der Umsetzung gesundheitsorientierter Führung aufgeführt [8].

\section{Zusammenfassung und Ausblick}

Zusammenfassend zeigt sich, dass Führungskräfte eine wichtige Funktion in der betrieblichen Gesundheitsförderung einnehmen können [19, 20, 43]. Sie beeinflussen den Gesundheitszustand der Beschäftigten direkt über ihre gesundheitsorientierte Mitarbeiterführung [13, $14,17,39]$ oder indirekt über den eigenen Gesundheitszustand [21] sowie die gesundheitsorientierte Selbstführung der Führungskräfte und Mitarbeitenden [14, 20, 25, 26, 39]. Auch wenn die aktuelle empirische Studienlage deutlich macht, dass gesundheitsorientierte Führung noch kein wissenschaftlich gut untersuchtes Konstrukt ist, so wird doch eine erhebliche Relevanz für Wissenschaft und Praxis erkennbar [35, 37]. Bisherige empirische Studienergebnisse geben Hinweise darauf, welche Chancen für die Betriebliche Gesundheitsförderung entstehen, wenn gesundheitsorientierte Führung in das Führungsleitbild, die Personalentwicklung wie auch das betriebliche Gesundheitsmanagement integriert und gefördert wird. Da empirische Studien zur Übertragbarkeit des Ansatzes gesundheitsorientierter 
Führung im virtuellen Arbeitskontext bislang noch nicht vorliegen [42], sollte zukünftige Forschung untersuchen, wie gesundheitsorientierte Führung in virtuellen Teams Anwendung findet. Insbesondere der HoL-Ansatz [12] wird als geeignetes Modell und Instrument für die weitere Forschung empfohlen.

\section{Fazit für die Praxis}

- Führungskräfte können in der betrieblichen Gesundheitsförderung von entscheidender Relevanz sein, denn mit ihrer Selbst- und Mitarbeiterführung sowie ihrer Vorbildfunktion können sie die Gesundheit der Mitarbeitenden auf verschiedenen Ebenen nachweislich beeinflussen.

- Aufgrund steigender gesundheitlicher Belastungsfaktoren in digitalisierten und flexibilisierten Arbeitswelten wie virtueller Teamarbeit wird die Implementierung gesundheitsorientierter Führung in das Betriebliche Gesundheitsmanagement angeraten.

- Die Anwendungsmöglichkeiten gesundheitsorientierter Führung in virtueller Teamarbeit müssen in der Praxis empirisch untersucht werden.

- Für die weitere Forschung gesundheitsorientierter Führung wird das HoL-Instrument (,health-oriented leadership") empfohlen.

\section{Korrespondenzadresse}

\section{llona Efimov, M.Sc.}

Zentralinstitut für Arbeitsmedizin und Maritime Medizin (ZfAM), Universitätsklinikum Hamburg-Eppendorf (UKE)

Seewartenstraße 10 | Haus 1 |, 20459 Hamburg, Deutschland

i.efimov@uke.de

Funding. Open Access funding enabled and organized by Projekt DEAL.

\section{Einhaltung ethischer Richtlinien}

Interessenkonflikt. I. Efimov, V. Harth und S. Mache geben an, dass kein Interessenkonflikt besteht.

Für diesen Beitrag wurden von den Autoren keine Studien an Menschen oder Tieren durchgeführt. Für die aufgeführten Studien gelten die jeweils dort angegebenen ethischen Richtlinien.
Open Access. Dieser Artikel wird unter der Creative Commons Namensnennung 4.0 International Lizenz veröffentlicht, welche die Nutzung, Vervielfältigung, Bearbeitung, Verbreitung und Wiedergabe in jeglichem Medium und Format erlaubt, sofern Sie den/die ursprünglichen Autor(en) und die Quelle ordnungsgemäß nennen, einen Link zur Creative Commons Lizenz beifügen und angeben, ob Änderungen vorgenommen wurden.

Die in diesem Artikel enthaltenen Bilder und sonstiges Drittmaterial unterliegen ebenfalls der genannten Creative Commons Lizenz, sofern sich aus der Abbildungslegende nichts anderes ergibt. Sofern das betreffende Material nicht unter der genannten Creative Commons Lizenz steht und die betreffende Handlung nicht nach gesetzlichen Vorschriften erlaubt ist, ist für die oben aufgeführten Weiterverwendungen des Materials die Einwilligung des jeweiligen Rechteinhabers einzuholen.

Weitere Details zur Lizenz entnehmen Sie bitte der Lizenzinformation auf http://creativecommons.org/ licenses/by/4.0/deed.de.

\section{Literatur}

1. Akin N, Rumpf J (2013) Führung virtueller Teams. Gruppendyn Organisationsberat 44:373-387

2. Bell BS, Kozlowski SW (2002) A typology of virtual teams: Implications for effective leadership. Group Organ Manag 27:14-49

3. Böhm SA, Bourovoi K, Brzykcy A et al (2016) Auswirkungen der Digitalisierung auf die Gesundheit von Berufstätigen: Eine bevölkerungsrepräsentative Studie in der Bundesrepublik Deutschland. Universität St. Gallen, St. Gallen

4. Breuer C, Hüffmeier J, Hertel G (2017) Vertrauen per Mausklick: Wie Vertrauen in virtuellen Teams entstehen kann. PERSONALquarterly 69:10-16

5. Coun M, Peters P, Blomme R (2019) Taking the leadership role: Understanding leadership across team and organizational boundaries in view of the changing employment relationship. J Leadersh Stud 12:65-68

6. Cree T, Kelloway EK (1997) Responses to occupational hazards: Exit and participation. J Occup Health Psychol 2:304-311

7. Echterhoff M (2011) Führung und Gesundheit: Welchen Einfluss nehmen Führungskräfte auf die gesundheitsförderlichen bzw. gesundheitsbelastenden Arbeitsbedingungen der Beschäftigten fü sich selbst wahr. In: Fakultät für Gesundheitswissenschaften. Universität Bielefeld, Bielefeld

8. Efimov I, Harth V, Mache S (2020) Health-oriented self- and employee leadership in virtual teams: a qualitative study with virtual leaders. Int J Environ Res Publ Health 17:6519

9. Eriksson A, Axelsson R, Axelsson SB (2011) Health promoting leadership-Different views of the concept. Work 40:75-84

10. European Agency for Safety and Health at Work (2016) Second European survey of enterprises on new and emerging risks (ESENER-2). Overview report: managing safety and health at work. European risk observatory. Publications Office of the European Union, Luxemburg

11. Franke F, Ducki A, Felfe J (2015) Gesundheitsförderliche Führung. In: Felfe J (Hrsg) Trends der psychologischen Führungsforschung. Hogrefe, Göttingen, S253-264
12. Franke F, Felfe J (2011a) Diagnose gesundheitsförderlicher Führung - Das Instrument "Healthoriented Leadership". In: Badura B, Ducki A, Schröder H, Klose J, Macco K (Hrsg) FehlzeitenReport 2011. Führung und Gesundheit: Zahlen, Daten, Analysen aus allen Branchen der Wirtschaft. Springer, Berlin, Heidelberg

13. FrankeF,FelfeJ(2011b) How does transformational leadership impact employees' psychological strain? Examining differentiated effects and the moderating role of affective organizational commitment. Leadership 7:295-316

14. Franke F, Felfe J, Pundt A (2014) The impact of health-oriented leadership on follower health: developmentand test of anewinstrumentmeasuring health-promoting leadership. Z Personalforsch 28:139-161

15. Gregersen S, Kuhnert S, Zimber A et al (2011) Führungsverhalten und Gesundheit - Zum Stand derForschung. Gesundheitswesen 73:3-12

16. Hoch JE, Kozlowski SW (2014) Leading virtual teams: hierarchical leadership, structural supports, and shared team leadership. J Appl Psychol 99:390-403

17. Horstmann D, Remdisch S (2016) Gesundheitsorientierte Führung in der Altenpflege. Bedeutung sozialer Belastungen und Ressourcen für die Gesundheit und das Commitment der Mitarbeiter. ZArb Organisationspsychol 60:199-211

18. Kaluza AJ, Boer D, Buengeler C et al (2020) Leadership behaviour and leader self-reported well-being:a review, integration and meta-analytic examination. Work Stress 34:34-56

19. Kelloway EK, Barling J (2010) Leadership development as an intervention in occupational health psychology. Work Stress 24(3):260-279. https:// doi.org/10.1080/02678373.2010.518441

20. Klug K,Felfe J, KrickA (2019) Caring for oneself or for others? How consistent and inconsistent profiles of health-oriented leadership are related to follower strain and health. Front Psychol 10:2456. https:// doi.org/10.3389/fpsyg.2019.02456

21. Köppe C, Kammerhoff J, Schütz A (2018) Leaderfollower crossover: exhaustion predicts somatic complaints via StaffCare behavior. J Manag Psychol 33:297-310

22. Kordsmeyer A-C, Mette J, Harth V et al (2019a) Arbeitsbezogene Belastungsfaktoren und Ressourcen in der virtuellen Teamarbeit. Zentralbl Arbeitsmed Arbeitsschutz Ergonomie 69:239-244

23. Kordsmeyer A-C, Mette J, Harth V et al (2019b) Gesundheitsorientierte Führung in der virtuellen Teamarbeit. Zentralbl Arbeitsmed Arbeitsschutz Ergonomie 70:76-82

24. Kranabetter C, Niessen C (2016a) How managers respond to exhausted employees. J Pers Psychol 15:106-115

25. Kranabetter C, Niessen C (2016b) Managers as role models for health: moderators of the relationship of transformational leadership with employee exhaustion and cynicism. J Occup Health Psychol 22:492-502

26. Krick A, Felfe J, Klug K (2019) Turning intention into participation in occupational health promotion courses? The moderating role of organizational, intrapersonal, and interpersonal factors. J Occup Environ Med 61:779-799

27. Kuoppala J, Lamminpää A, Liira J et al (2008) Leadership, job well-being, and health effects - a systematic review and a meta-analysis. JOccup Environ Med 50:904-915

28. Liao C (2017) Leadership in virtual teams: a multilevel perspective. Hum Res Manage Rev 27:648-659 
29. Mache S, Harth V (2019) Digitale Transformation in der Arbeitswelt und psychische Gesundheit. Zentralbl Arbeitsmed Arbeitsschutz Ergonomie. https://doi.org/10.1007/s40664-019-00369-3

30. Meyer M, Maisuradze M, Schenkel A (2019) Krankheitsbedingte Fehlzeiten in der deutschen Wirtschaft im Jahr 2018 - Überblick. In: Badura B, Ducki A, Schröder H, Klose J, Meyer M (Hrsg) Fehlzeiten-Report 2019. Digitalisierung gesundes Arbeiten ermöglichen. Springer, Berlin, Heidelberg, S413-477

31. Montano D, Reeske A, Franke F et al (2017) Leadership, followers' mental health and job performance in organizations: a comprehensive meta-analysis from an occupational health perspective. J Organiz Behav 38:327-350

32. Montano D, Reeske-Behrens A, Franke F (2016) Psychische Gesundheit in der Arbeitswelt Führung. Bundesanstalt für Arbeitsschutz und Arbeitsmedizin, Dortmund

33. Oreg S, Berson Y (2011) Leadership and employees' reactions to change: The role of leaders' personal attributes and transformational leadership style. Personnel Psychol 64:627-659

34. Panteli N, YalabikZY, Rapti A (2019) Fostering work engagement in geographically-dispersed and asynchronous virtual teams. Inf Technol People 32:2-17

35. Rigotti T, Holstad T, Mohr G et al (2014) Rewarding and sustainable health-promoting leadership. Bundesanstalt für Arbeitsschutz und Arbeitsmedizin, Dortmund

36. Robelski S, Harth V, Mache S (2018) Anforderungen an Führung im Kontext flexibler Arbeitswelten. Wie kann Führung gesundheitsförderlich gestaltet werden? Zentralbl Arbeitsmed Arbeitsschutz Ergonomie 68:118-124

37. Rudolph CW, Murphy LD, Zacher H (2020) A systematic review and critique of research on "healthyleadership". Leadersh Q 31:1-21

38. RW ${ }^{3}$ Culture Wizard (2018) 2018 trends in highperforming global virtual teams

39. Maria SA, Wolter C, Gusy B et al (2019) The impact of health-oriented leadership on police officers' physical health, burnout, depression and wellbeing. Policing: A J Policy Pract 13:186-200

40. Schwarzmüller T, Brosi P, Welpe IM (2017) Führung 4.0 - Wie die Digitalisierung Führung verändert. In: Hildebrandt A, Landhäußer W (Hrsg) CSR und Digitalisierung.DerdigitaleWandelalsChance und Herausforderung für Wirtschaft und Gesellschaft. Springer, Berlin, Heidelberg, S617-628

41. Skakon J, Nielsen K, Borg V et al (2010) Are leaders' well-being, behaviours and style associated with the affective well-being of their employees? A systematic review of three decades of research. Work Stress 24(2):107-139. https://doi.org/10. 1080/02678373.2010.495262

42. Staar H, Gurt J, JanneckM (2019) Gesunde Führung in vernetzter (Zusammen-) Arbeit - Herausforderungen und Chancen. In: Badura B, Ducki A, Schröder H, Klose J, Meyer M (Hrsg) FehlzeitenReport 2019. Digitalisierung - gesundes Arbeiten ermöglichen. Springer, Berlin, Heidelberg, S 217-236

43. Stilijanow U (2012) Führung und Gesundheit. In: Lohmann-Haislah A (Hrsg) Stressreport Deutschland 2012. Psychische Anforderungen, Ressourcen und Befinden. Bundesanstalt für Arbeitsschutz und Arbeitsmedizin, Dortmund, S 123-128

44. Vincent-Höper S, Stein M (2019) The role of leaders in designing employees' work characteristics: validation of the health-and development- promoting leadership behavior questionnaire. Front Psychol 10:1-15

45. Wegge J, Shemla M, Haslam SA (2014) Leader behavior as a determinant of health at work: specification and evidence of five key pathways. Ger JHum Resour Manag 28(1-2):6-23. https://doi. org/10.1177/239700221402800102 